\title{
Overview of Wireless Access in Vehicular Environment (WAVE) Protocols and Standards
}

\author{
Shereen A. M. Ahmed ${ }^{1 *}$, Sharifah H. S. Ariffin ${ }^{2}$ and Norsheila Fisal ${ }^{3}$ \\ 'Ph.D Student, UTM; Shereen_malek@yahoo.com \\ ${ }^{2}$ Associated Professor, UTM; sharifah@fke.utm.my \\ 3Professor, Head of UTM MIMOS; Sheila@fke.utm.my
}

\begin{abstract}
Institute of Electrical and Electronics Engineers (IEEE) series of standards for Wireless Access in Vehicular Environment (WAVE) is currently considered as the most promising technology for vehicular networks. It aims to support interoperability and robust safety communications in a vehicular environment. The first trial version of WAVE standard was released in 2006, Most of the published review studies were done on the old trail versions. This study reviews the latest released version of the IEEE WAVE standard, reflects the state of art in WAVE technology, and presents the status of each standard in the IEEE 1609 series of standards. IEEE 1609 series contains four standards that are under development, three are published and one is already withdrawn. Then the study discusses the technical details of IEEE 1609.4 for multi-channel operation and highlights the new features of WAVE Media Access Control (MAC) layer. The study also describes the services and protocols that are provided by IEEE 1609.3 as a network service standard. Frame format that is used in standards are also exposed.
\end{abstract}

Keywords: VANET, IEEE WAVE, IEEE 1609.3, IEEE 1609.4, Intelligent Transportation Systems ITS, WSMP.

\section{Introduction}

Since early 1990s efforts have been increased to integrate technology with transportation systems to build "smart road" that are capable of guiding vehicles to improve the traffic safety and conditions [1,2]. Effective use of such system would have great impact on the overall transportation system performance, and it is expected to decrease the probability of traffic accidents and loss of life, provide traffic management in term of vehicle traffic flow and coordination, and deliver infotainment applications to the passenger. Furthermore, it can help in reducing pollution and conserve fuel $[3,4]$. Vehicular Ad-hoc Networks (VANET) is a kind of large scale Mobile ad-hoc Networks (MANET) that turn moving cars into wireless nodes. VANET is characterized by its high mobility of nodes, wide range of relative speeds between vehicles and real time nature of applications [5]. However, in VANETs, nodes tend to move in an organized pattern (e.g. Streets), thus, portability issues like power consumption and antennas are not significant problems. Generally, vehicular network system components may consist of an On-Board Equipment (OBE) which is a network device located in the moving vehicle and connected to both wireless network and to in-vehicle network, Road-Side Equipment (RSE), which can be described as a device installed in the side-road infrastructure (e.g. Light pole and road signs) that connects the moving vehicle to the access network which in turn is connected to the core network [3]. Vehicular networks would potentially have two main types of communications [6].

1 Vehicle to Vehicle (V2V) communication: where communications between vehicles mainly include safety applications and traffic Management applications. The most important is safety applications, which are time sensitive and high priority applications. According to

*Corresponding author:

Shereen A. M. Ahmed (Shereen_malek@yahoo.com) 
Wireless Access in Vehicular Environment (WAVE) Standards, OBEs can communicate with each other over a fixed radio channel called a Control Channel $(\mathrm{CCH})$, WAVE standard provides a unique communication mode called WAVE Short Message Protocols (WSMP) mode, which enable the exchange of messages in a rapidly varying radio frequency $(\mathrm{RF})$ environment where low latency is an important objective. WSMP is used to facilitate communication among vehicles in case of safety applications $[1,6]$.

2 Vehicle to Infrastructure (V2I) Communication: OBEs and RSE can exchange data and control information with each other, each RSE is connected to the wide area network using any type of wire or wireless technology (Cellular, WiMAX). Internet Protocol version 6 (IPv6) stack is included to support the more traditional and less demanding applications, such as internet applications, which are required to attract the private investments and reduce the cost of system implementation [6]. VANET applications can be divided into the following categories 1- Road safety applications, which include sharing information among vehicles or between vehicles and roadside units to ensure safety, and considered as the best way to reflect the value of intelligent transportation system, such applications may include: collision warning applications, and emergency vehicle warning applications. 2- Traffic management applications that provide local information and update maps so as to improve traffic efficiency and coordination. 3- Infotainment applications that involve traditional Internet access like community services and commercial services [3].

\section{WAVE Protocols and Model}

The Institute of Electrical and Electronics Engineers (IEEE) 1609 Family of Standards for WAVE, defines the architecture and standardizes set of services and interfaces that enable secure wireless communication and physical access for high speed (up to $27 \mathrm{MB} / \mathrm{s}$ ), short range (up to $1000 \mathrm{~m}$ ), and low latency wireless communication in the vehicular environment $[7,8]$.

As indicated in table (1), IEEE1609 series standard consists of three published standards and four unpublished standards under development. WAVE Physical Layer is based on the IEEE 802.11, however, because of the operating environment of vehicular networks; an amendment of the standard is made which is known as IEEE 802.11p $[2,6]$.
Figure 1 compares WAVE protocol stack to the Transmission Control Protocol (TCP) stack, WAVE stacks model have the same physical and data link layers, but it differs in the network and transport layers. Security layer does not fit in the TCP protocol stack. In general, IEEE 802.11p and IEEE 1609.4 are used to describe the physical and the Media Access Control (MAC) layer of the system respectively. While IEEE 1609.3, with its two supported stacks (e.g. Internet Protocol version 6 (IPv6) stack and WAVE Short Message Protocol (WSMP) stack) is used to describe a network layer and transport layer of the TCP stack. Security functions and services are described by an IEEE 1609.2 standard protocol. The next sections discuss on each layer.

\section{WAVE Physical Layer}

WAVE physical layer has been described by IEEE $802.11 \mathrm{p}$ standard protocol WAVE is relies on IEEE 802.11a Orthogonal Frequency Division Multiplexing (OFDM) mechanism to support different data rates that are determined by coding rate and modulation type. The $75 \mathrm{MHz}$ bandwidth in $5.9 \mathrm{GHz}$ spectrum is divided into 7 smaller operation channels each of $10 \mathrm{MHz}$ bandwidth [10].

As illustrated in Figure 2, Channel 178 is used as Control Channel (CCH) while channel 174, 176, 180 and 182 are used as Service Channel (SCH). Channel 184 and 172 are left for future use. Multi physical layer device will typically work in $\mathrm{CCH}$ and at least one SCH [7]; while single Physical layer device may switch between $\mathrm{CCH}$ and SCH. (See section 3.1) In order to accommodate with this limited capability of the single physical layer device, synchronization is required to ensure that all WAVE devices monitor the $\mathrm{CCH}$ at the same time interval [11]. As soon as a group of WAVE devices are synchronized they can simultaneously monitor and/or utilize the control channel. In [1] Current WAVE standard follows a simple synchronization mechanism in which all WAVE devices align their radio resource to a globally accurate clock every time period.

\subsection{Channel Access Schemes}

Unlike other Local Area Networks (LANs) technologies, WAVE devices will be required to monitor two channels, Control Channel (CCH) and Service Channel $(\mathrm{SCH})[12,13], \mathrm{CCH}$ is used for service announcement and WAVE short messages that is used by safety application, while SCH carries the data of the IPv6 stack. WAVE standard also provides two sets of devices, either cheap 
Table 1. IEEE WAVE Family Standards Description [9]

\begin{tabular}{|c|c|c|}
\hline WAVE Standard & Usage & Description \\
\hline IEEE P1609.0 & Architecture & $\begin{array}{l}\text { Describes the architecture and service necessary for } \\
\text { multi-channel WAVE devices }\end{array}$ \\
\hline IEEE P1609.2 & $\begin{array}{l}\text { Security Services for Applications and } \\
\text { Management Messages }\end{array}$ & $\begin{array}{l}\text { Covers methods for securing WAVE management } \\
\text { messages and application messages, It also describes } \\
\text { administrative functions necessary to support the } \\
\text { core security functions }\end{array}$ \\
\hline IEEE 1609.3-2010 & Networking Service & $\begin{array}{l}\text { Describes standard messages that support higher } \\
\text { layer communication stacks, including TCP/IP }\end{array}$ \\
\hline IEEE 1609.4-2010 & Multi-Channel Operation & $\begin{array}{l}\text { Describes various standard message formats for } \\
\text { DSRC applications at } 5.9 \mathrm{GHz}\end{array}$ \\
\hline IEEE P1609.5 & Communication Manager & $\begin{array}{l}\text { Defines communication management services in } \\
\text { support of wireless connectivity among vehicle- } \\
\text { based devices, and between fixed roadside devices } \\
\text { and vehicle-based devices }\end{array}$ \\
\hline IEEE 1609.11-2010 & $\begin{array}{l}\text { Over-the-Air Electronic Payment Data } \\
\text { Exchange Protocol }\end{array}$ & $\begin{array}{l}\text { Defines a basic level of technical interoperability for } \\
\text { electronic payment equipment, i.e. On board unit } \\
(\mathrm{OBU}) \text { and roadside equipment (RSE) using DSRC }\end{array}$ \\
\hline IEEE P1609.12 & Identifier Allocations & $\begin{array}{l}\text { Specifies allocations of WAVE identifiers defined in } \\
\text { the IEEE } 1609 \text { series of standards }\end{array}$ \\
\hline
\end{tabular}
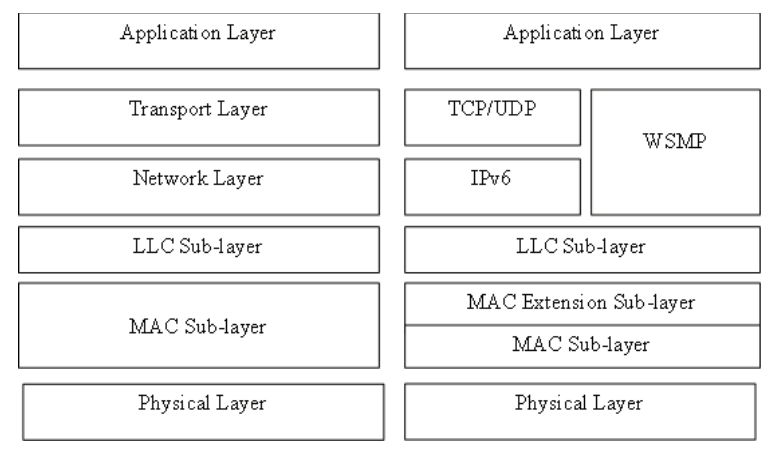

Figure 1. Comparison of WAVE Protocol Stack with TCP Protocol Stack, as indicated, WAVE security layer does not fit into the TCP protocol stack.

\begin{tabular}{|c|c|c|c|c|c|c|}
\hline CHI72 & CH174 & CH176 & CH178 & CH180 & CH182 & CH184 \\
\hline
\end{tabular}

Figure 2. WAVE radio channels arrangement.

single physical Layer device which is not capable of simultaneous operation on multiple radio channels, or, multiphysical Layer Device which exchange data on one channel (e.g. SCH) while at least actively listen to a second channel (e.g. $\mathrm{CCH}$ ) [14].

Figure 3 illustrates examples of variations WAVE devices Physical layer operation. Switching device can be

\begin{tabular}{|c|c|c|c|c|c|}
\hline $\mathrm{CCH}$ & $\mathrm{SCH}$ & $\mathrm{CCH}$ & $\mathrm{SCH}$ & $\mathrm{CCH}$ & $\mathrm{SCH}$ \\
\hline \multicolumn{6}{|c|}{ (a) } \\
\hline \multicolumn{6}{|c|}{$\mathrm{CCH}$} \\
\hline \multicolumn{6}{|c|}{ SCH } \\
\hline
\end{tabular}

Figure 3. Examples of WAVE devices physical layer operation (a) Single Physical Layer Device alternatively switching between Control Channel $(\mathrm{CCH})$ and Service Channel (SCH). (b) Multi Physical layers Device each physical layer continuously monitors either $\mathrm{CCH}$ or $\mathrm{SCH}$.

defined as a device with at least one physical layer that is capable of switching between channel (e.g. SCH and $\mathrm{CCH})$. Control Channel interval (CCHI) and Service Channel interval (SCHI) are the period of times during which the WAVE device may visit $\mathrm{CCH}$ and $\mathrm{SCH}$, respectively [14].

\subsection{Single Physical Layer Device Access Scheme}

As explained by Figure 4, and based on the service request, a single physical device can get access to the channel in one of the following access schemes:

Continuous Access: where single physical device will operate on $\mathrm{CCH}$ only. Alternating Access: where single 
physical device access $\mathrm{SCH}$ in $\mathrm{SCH}$ interval and $\mathrm{CCH}$ in $\mathrm{CCH}$ interval. Immediate or extended Access: where single physical device access $\mathrm{SCH}$ during $\mathrm{SCH}$ interval and $\mathrm{CCH}$ interval. To override the immediate and extended $\mathrm{SCH}$ access, $\mathrm{CCH}$ access during $\mathrm{CCH}$ and/or $\mathrm{SCH}$ interval is provided.

\subsection{Multi-physical Layer Device Access Scheme}

All channel access options that are available for a single physical device can be used for multi physical layer device access.

In a scenario of multi physical layer device with at least on switching device, and as illustrated in Figure 5, physical layer 1 is switching between its $\mathrm{CCH}$ and $\mathrm{SCH} 1$ while Physical layer 2 is inactive, as soon as physical 1 detects a WAVE Service Advertisement (WSA) (will be further detailed in section 5) during $\mathrm{CCH}$ interval it passes it to the WAVE Management Entity (WME) (will be detailed in 5). When the higher layer requests access to the announced service, WME will trigger the MAC to assign the $\mathrm{SCH}, \mathrm{MAC}$ layer will respond by assigning $\mathrm{SCH} 2$ of physical layer 2. When the higher layer requests termination of the service, WME will terminate $\mathrm{SCH} 2$ of Physical layer 2 and the MAC returns physical layer 2 to its inactive state[14].

\section{WAVE MAC Layer}

MAC layer functions are defined by the IEEE 1906.4 standard. Figure 6 is the basic reference model relevant to the standard. In fact, WAVE captures the fundamentals of IEEE 802.11e Enhanced Distributed Channel Access (EDCA) Paradigm and extends it to cover two channels operations. A typical reference model consists of two planes, data plane and management plane. Data plan provides data services such as inbound and outbound high layers data, while the management plane performs the management command such as synchronization and channel access. Physical Layer Management Entity (PLME) is the management entity that provides management function to the physical layer. IEEE 1609.4 specifies extension to 1EEE 802.11 MAC sub layer Management Entity (MLME) to provide channel coordination, which is required when there are one or more switching devices with concurrent alternating operation on $\mathrm{CCH}$ and $\mathrm{SCH}$, so that data packets are transmitted on the proper channel

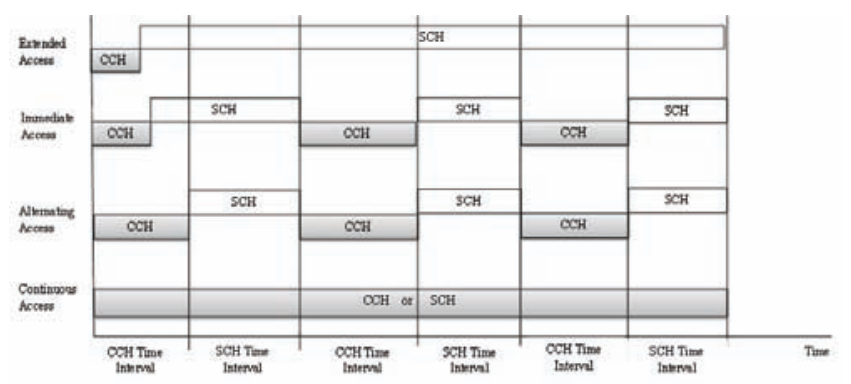

Figure 4. Types of WAVE Channel Access schemes: Continuous Access, Alternating Access, Immediate Access, and Extended Access.

at the right time. MLME is providing services for both data plane and management plane, data plane services which are performed by MLME include: channel coordination, channel routing and user priority while management services are: multi-channel synchronization, channel access, Vendor specific actions frames, Management Information Base (MIB) maintenance and readdressing. Three types of frames are allowed in WAVE: 1- Control frames which are used as per IEEE 802.11 standard. 2- Management frames which are either Time Advertisement frames (TA), used to advertise time synchronization information, or, Vendor specific Action (VSA) frames. Management frames can be transmitted on $\mathrm{CCH}$ or SCH. 3- Data frames which might be WSMP data or IP datagram, WSMP frames which containing WAVE Service Messages (WSM) may get transmitted on $\mathrm{CCH}$ or $\mathrm{SCH}$, However, IP datagram can only be transmitted on SCH. Figure 6 describes the internal architecture of Multi-Channel operation MAC, Figure 6 contains both $\mathrm{CCH}$ and $\mathrm{SCH}$. Queues are provided to perform access of the prioritized data [15]. When data is received from Logical Link Control (LLC), the channel router checks the ethertype field in the header of the data, if it is WSMP datagram, then it should be routed to the proper queue based on channel identifier and data priority. However, IP datagram transmission is slightly different, all IP data should be registered with the transmitter profile in the MIB which contains $\mathrm{SCH}$ number, power level and the adaptable status of power level and data rate. When LLC passes an IPv6 datagram to the channel router, it routs the datagram to the data buffer that corresponds to the specified $\mathrm{SCH}$. Those procedures are allowing the higher layer to control the transmission parameters of the physical layers. Channel selector carries out many functions such as, drops the data when the channel is no longer valid. $[1,7]$. 


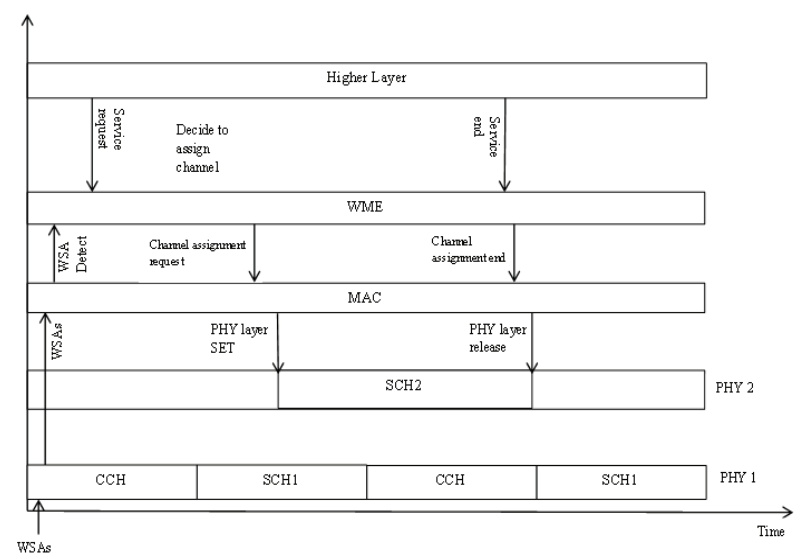

Figure 5. Illustration of Example for Multi-Physical (PHY) layers Device internal operation. ${ }^{*} \mathrm{CCH}$ : Control Channel.

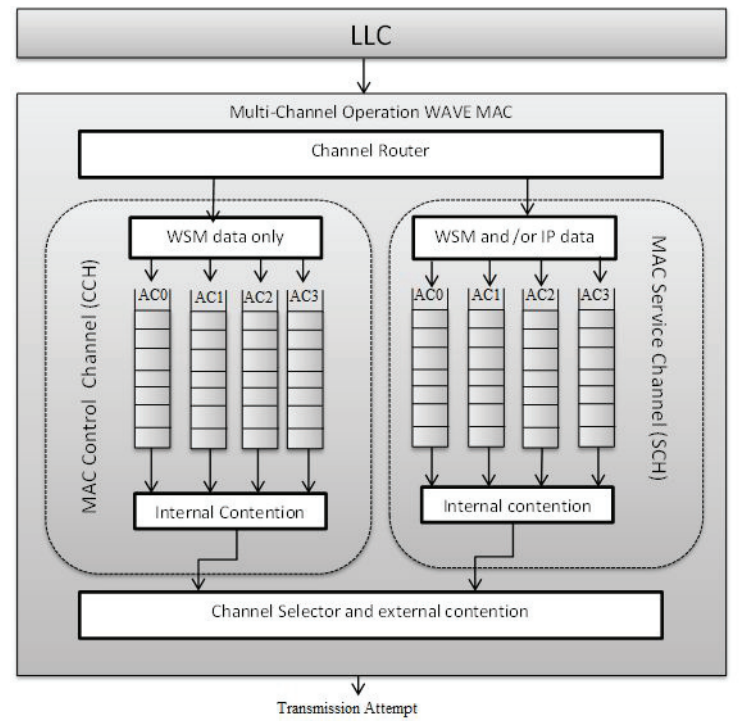

Figure 6. Description for internal architecture of multichannel MAC Layer WAVE device.

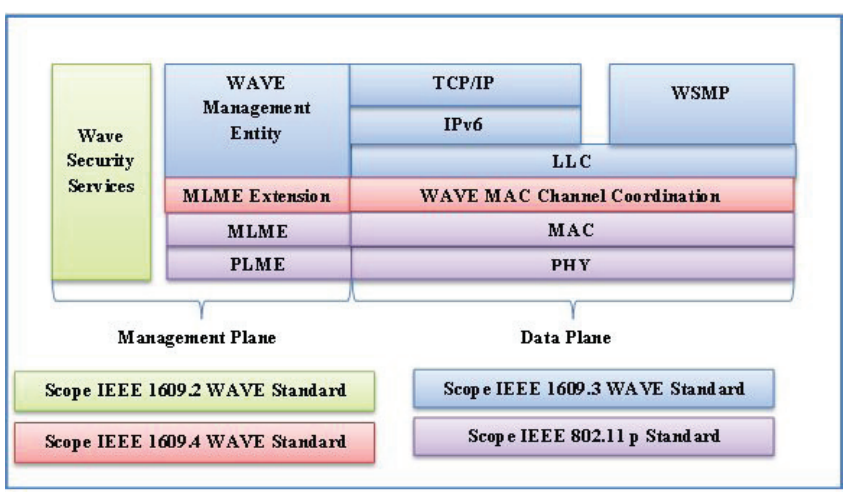

Figure 7. WAVE protocol stack indicating the standard that covers each set of layers. ${ }^{*}$ IPv6: Internet Protocol version 6.

\section{WAVE Network Services}

As indicated in Figure 7, the network services are defined by the IEEE 1609.3 Standard protocol and describe data plane and management plane services. Two types of WAVE devices are defined: Provider device which is the device transmitting WAVE Service Advertisement (WSA) to indicate its availability for data exchange on one or more SCHs, provider WAVE device is the sender of WSA and it is the initiator of the service. User device is the device that monitoring for received WSAs, with a possibility of participation in the $\mathrm{SCH}$ data exchange, user device is the receiver of the WSA and joiner of the service. WAVE device may assume one, both or neither device [14].

\subsection{Data Plane Services}

As illustrated by Figure 7, network service data plane support two protocol stacks, WSMP protocol stack and IPv6 protocol stack.

\subsubsection{WAVE Short Message Protocol (WSMP)}

WAVE Short Message Protocol (WSMP) is a WAVE network layer unique protocol to support high priority and time sensitive communication. Upon reception of WAVE Short Massage (WSM) data unit form upper layers, WSMP generate WSMP header to be included in the received unit, then make packet transmission request to LLC, LLC set the ethertype field value to encapsulate the packet and pass the data to the lower layers. When the LLC receives MAC data unit, it checks the ethertype field value, then delivers it to either IPv6 or WSMP stack [14].

\subsubsection{Internet Protocol Version 6 IPv6}

WAVE standard supports UDP/TCP/IP protocol suite. IP traffic is sent and received through the LLC sub layer.

\subsection{Management Plane Services}

\subsubsection{WAVE Management Entity (WME)}

Provides WAVE networking management services functions, such as processing the service requests for the higher layers, providing channel assignments, monitoring WAVE service Announcements, configure IPv6 using data received from other WAVE devices, and maintains MIB.

\subsubsection{Management Information Base (MIB)}

MIB is information base, includes channel related info (e.g. Transmitter profile table) and timing info. MIB also contains 
the system configuration information and system status information, System configuration information includenumber of channels supported, advertiser Identifier, registration port, and WMS max length. While status information provide group of status tables namely: Provider Service Request Table (PSRT), User Service Request Table (USRT), CCH Service Request Table (CSRT) and WSM Service Request Table (WSRT). PSRT is table located in MIB and maintains provider information such as Provider Service Identifier (PSID) of the registered application, IPv6 address, port number, and so on. USRT is also located in MIB and maintaining information such as user PSIDs, advertiser identifier, link quality, channel number and so on, CSRT contains: $\mathrm{CCH}$ interval, request priority, and request status, and WSRT contains PSIDs of the registered applications [14].

\subsubsection{WAVE Services Request}

WAVE service may be either added, changed or deleted, in facts, there are six types of WAVE service requests Provider service request: when higher layer entity generate a provider service request, the WME accept the request, assign $\mathrm{SCH}$ access and triggers the MLME to start generating WSAs.

\subsubsection{User Service Request}

When higher layer generate user service request, while WME mentoring for WSAs, it detects matching available service and assign SCH access. WSM service request: when a higher layer generates a WSM service request indicating its willing to receive WSM of a particular PSID, the WME accept the request and ongoing of its monitoring, it delivers any received WSMs with matching PSID to the requested higher entity.

\subsubsection{CCH Service Request}

When a higher layer needs ongoing $\mathrm{CCH}$ access during a particular interval for a WSM activity or WSA reception it generate $\mathrm{CCH}$ service request, which will be considered by WME.

\subsubsection{Management Data Service Request}

When another management data entity wish to have vendor specific action frames (VSA), WME accept the request, assign $\mathrm{SCH}$ and trigger the MLME to generate VSA frame.

\subsubsection{Timing Advertisement Service Request}

when another management entity generate timer advertisement service request it indicates the WME its intent to transmit TA frame, WME accept the request, assign $\mathrm{SCH}$ or $\mathrm{CCH}$, and trigger the MLME to generate TA frame [14].

\section{WSM Frame Format:}

WSM frame is data structure used to send WAVE short messages frames.

Figure 8 is the WSM frame that has the following fields [14] WSMP Version field shows the version of the WAVE protocol, PSID Provider Service Identifier field, Channel Number field which defines the channel that is used for communication, Data Rate field specifies the data rate used in transmission, WAVE element ID field which represents WSMP header, WAVE Length field which determines length of the data field, and WSM Data field contains the payload data e.g. "Hello World".

\section{WSA Frame Format:}

WSA frame is data structures send by provider to announce the availability of service. Figure 9 shows the frame format of WSA.

\section{Provider Service Identifier PSID}

For the application to get access to the WAVE service, it should be registered with its unique Provider Service Identifier (PSID), PSID is a numerical field used by IEEE1609 series to identify a particular application. Applications intended to exchange data with vehicles would be installed in the Road side devices. The WAVE provider devices use PSID in its announcement messages to indicate that, a certain application is provided by this device. On the other hands, as the vehicle passing the road side device, user devices, which may host such application, upon reception of

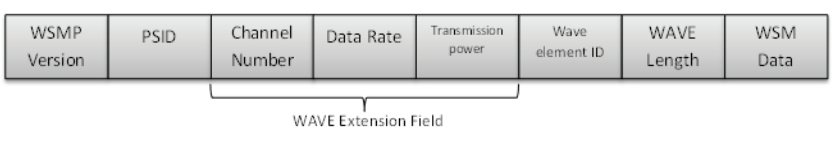

Figure 8. Data structure of WAVE Service Message (WSM) frame format.

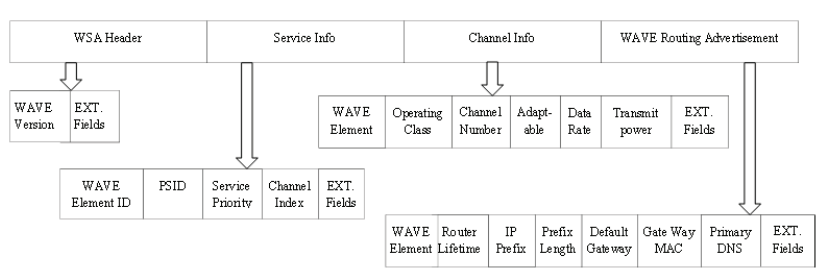

Figure 9. WAVE Service Advertisement (WSA) frame format. 
such announcement, compares to check if there is a match between the PSIDs in the announcements and PSIDs in its own tables, then the vehicle establish communication with that road side unit. However, the same example is applicable in case of vehicle to vehicle communications [16].

\section{Conclusion}

Table (2) describes the status of each standard in WAVE family. IEEE 1609 series standards consist of the seven standards. IEEE 1609.1 Resource manager - which was used to describe the application layer- and IEEE 1609.2- 2006 for Security issues, both have been withdrawn and currently the standard society is developing the new version of IEEE 1609.2 for security as well as IEEE 1609.0 for Architecture and IEEE 1609.5 for communication Manager. However, the new versions of IEEE 1609.4 for multichannel operation and IEEE 1609.3 for Network services already release [9]. This study focuses on the general ITS system architecture, and technical description of IEEE 1609.3 and IEEE 1609.4. IEEE 1609.4-for multichannel operation - is designed to enable upper layer to control data transfers across multiple channels, without requiring knowledge of physical layer parameters. This study describes internal MAC multi-channel operation and channel access schemes that provided by the IEEE 1609.4 standard. The study also defined the services that provided by IEEE 1609.3 for network services. IEEE 1609.3 supports high-rate, low-latency communications between WAVE devices. IEEE 1609.3 standard is consistent with the vehicle-to-roadside and vehicle-to-vehicle communications needs of various ITS architectures. IEEE 1609.3 standard supports two protocol stack, WSMP which is used to send WAVE short messages and IPv6 protocol stack for traditional internet data transmission. Two types of channel are defined $\mathrm{CCH}$ which is used for management frame and WSM only, while Service Channel SCH can send both IP data and WSM messages. WAVE protocol stack as its description allows researcher to implement transport layer protocol on top of it but care should be taken not to compromise interoperability with other WAVE devices, other challenges like routing, forwarding, multi-home protocols and data aggregation is still open and researchers are encourage to create effort on them $[7,14]$.

\section{Acknowledgement}

The authors would like to thank all those who contributed toward making this $\backslash$ research successful. The authors wish to express their gratitude to Ministry of Higher Education (MOHE), Malaysia Research Management Center (RMC) for the sponsorship, and Telematic Research Group (TRG), Universiti Teknologi Malaysia for the financial support and advice of this project. (Vot number Q.J130000.2623.08J19)

\section{References}

1. Morgan Y L (2010). Managing DSRC and WAVE Standards Operations in a V2V Scenario, International Journal of Vehicular Technology, vol 2010.

Table 2. State of Art for IEEE WAVE Standards Series

\begin{tabular}{lll}
\hline WAVE Standard & Usage & Development Status \\
\hline IEEE P1609.0 & Architecture & Under Development \\
IEEE 1609.1-2006 & Resource Manager & Withdrawn \\
IEEE P1609.2 & Security Services for Applications and & -Version1 IEEE1609.2-2006 \\
& Management Messages & Withdrawn \\
IEEE 1609.3-2010 & Networking Service & -Version 2 under Development \\
IEEE 1609.4-2010 & Multi-Channel Operation & -Version1 Published 2007 \\
& & -Version2 published 2010 \\
IEEE P1609.5 & Communication Manager & -Version 1 Published 2006 \\
IEEE 1609.11-2010 & Over-the-Air Electronic Payment Data & - Version 2 Published 2010 \\
IEEE P1609.12 & Exchange Protocol & Under Development \\
\hline
\end{tabular}


2. Hartenstein H, and K P Laberteaux (2008). A tutorial survey on vehicular ad hoc networks, IEEE Communications Magazine, vol 46(6), 164-171.

3. Karagiannis $G$ et al. (2011). Vehicular networking: a survey and tutorial on requirements, architectures, challenges, standards and solutions, IEEE Communications Surveys \& Tutorials, vol 13(4), 584-616.

4. Alsabaan M et al. (2012). Vehicular networks for a greener environment: a survey, IEEE Communications Surveys \& Tutorials, vol PP(99), 1-17.

5. Grafling S, Mahonen P et al. (2010). Performance evaluation of IEEE 1609 WAVE and IEEE 802.11p for vehicular communications, 2010 Second International Conference in Ubiquitous and Future Networks (ICUFN).

6. Uzcategui R, and Acosta-Marum G (2009). Wave: a tutorial, IEEE Communications Magazine, vol 47(5), 126-133.

7. 1609.4-2010 - IEEE Standard for Wireless Access in Vehicular Environments (WAVE)--Multi-channel operation, 1-89.

8. Eichler S (2007). Performance evaluation of the IEEE 802.11p WAVE Communication Standard, 2007. VTC-2007 Fall. 2007 IEEE 66th Vehicular Technology Conference.

9. (RITA), R.a.I.T.A. ITS Standards Fact Sheets, Standards development statuses as of January 16, 2013. 2013.

10. Jiang D, and Delgrossi L (2008). IEEE 802.11p: Towards an International Standard for Wireless Access in Vehicular Environments, VTC Spring 2008, IEEE Vehicular Technology Conference, 2036-2040.
11. Ghosh S, Kundu A et al. (2011). Implementation challenges of time synchronization in vehicular networks, Recent Advances in Intelligent Computational Systems (RAICS), 2011 IEEE. 2011.

12. Di Felice $\mathrm{M}$ et al. (2010). On the impact of multi-channel technology on safety-message delivery, 2012 21st International Conference on Computer Communications and Networks (ICCCN).

13. Guo J et al. (2012). An adaptive and reliable MAC mechanism for IEEE 1609.4 and 802.11p VANETs, 2012 15th International Symposium on Wireless Personal Multimedia Communications (WPMC)

14. 1609.3-2010 - IEEE Standard for Wireless Access in Vehicular Environments (WAVE) - Networking Services, 1-144.

15. I.A., IEEE Standard for Information Technology (2005). Telecommunications and Information Exchange Between Systems - Local and Metropolitan Area Networks - Specific Requirements Part 11: Wireless LAN Medium Access Control (MAC) and Physical Layer (PHY) Specifications Amendment 8: Medium Access Control (MAC) Quality of Service Enhancements. IEEE Std 802.11e-2005 (Amendment to IEEE Std 802.11, 1999 Edition (Reaff 2003), 1-189.

16. Weil T (2009). Service management for ITS using WAVE (1609.3) Networking, 2009 IEEE GLOBECOM Workshops, $1-6$. 